\title{
Challenges and perspectives in the development of paper-based lateral flow assays
}

\author{
Surasak Kasetsirikul ${ }^{1,2}$, Muhammad J. A. Shiddiky ${ }^{1,3}$, Nam-Trung Nguyen ${ }^{2, *}$ \\ ${ }^{1}$ Queensland Micro and Nanotechnology Centre (QMNC), Griffith University Nathan Campus, \\ Nathan, Queensland 4111, Australia \\ ${ }^{2}$ School of Engineering and Built Environment, Griffith University Nathan Campus, Nathan, \\ Queensland 4222, Australia \\ ${ }^{3}$ School of Environment and Science, Griffith University Nathan Campus, Nathan, Queensland \\ 4111, Australia
}

*Corresponding author: Nam-Trung Nguyen (nam-trung.nguyen@griffith.edu.au)

\begin{abstract}
$\underline{\text { Abstract }}$
Lateral flow assays (LFAs) have been introduced and developed over the last half century. This technology is widely used as a tool for diagnosis in several fields such as environment, food quality and healthcare. Point-of-care (POC) diagnosis using LFAs has been attracting attention of the research community, particularly aiming for the development of a platform that can evaluate of biological markers in bodily fluids such as saliva and urine. The existence of a disease or the pregnancy can be determined by a test device, before further investigation and medical treatment. LFAs make use of a disposable test strip, which can provide diagnosis result on the spot within minutes. Thus, LFAs is a promising alternative of preliminary diagnosis for laboratory instruments that are costly, time-consuming and require trained personnel. This paper includes a brief overview of the conventional LFAs: material selection based on its roles and characteristics, working principles, fundamentals, applications, and design criteria. We mainly discuss the technical challenges in both engineering and biochemical aspects and recommends possible solutions. We identify current research trends and provide perspectives of advanced technologies for enhancing assay performance.
\end{abstract}

Keywords: Paper based microfluidics, lateral flow assays, point-of-care diagnosis 


\section{Introduction}

Recently, the need for low-cost medical diagnostic devices has been increasing, particularly in developing countries due to limited facilities and lack of qualified medical personnel $[1,2]$. The gold standards of medical diagnosis such as polymerase chain reaction (PCR) and enzyme-linked immunosorbent assay (ELISA) are traditionally based on lab-bench protocols, well-trained personnel and well-maintained diagnostics labs [3, 4]. Moreover, advanced medical diagnosis can detect a disease in the initial stage, leading to early and successful treatment. However, some diseases require dedicated and large instruments to be diagnosed, resulting in high operation cost, delay of treatment and inaccessibility, particularly in a remote areas [5, 6]. Consequently, point-of-care (POC) diagnostic device is a well-suited alternative for replacing laboratory-based equipment and skilled staff for relatively simple diagnosis [7].

The World Health Organization (WHO) provides seven major guidelines for the development of POC devices summarised in the acronym "ASSURED”: (1) Affordable: reasonable prices for settings composed of population at risk of infection, (2) Sensitivity: few false negative and lower limit of detection, (3) Specificity: few false-positives (4) User friendly: a few days of training and ease to use (5) Rapid and Robust: acceptable waiting time, long shelf lives without refrigerators and high throughput (6) Equipment-free: self-powered sources, on-site analysis, easily disposable, and easy sample handling (7) Deliverable: a portable or hand-held device [8-10]. Meeting these WHO guidelines, paper-based devices have been attracting extensive attention, especially because of their capability to be used in rural and limited-resource environments [11, 12].

Paper-based devices are cost effective, disposable and can be used with different biomolecules. Paper-based devices are especially simple because of the power-free fluid transport by capillary action $[11,13]$. Paper-based materials have been used for a range of applications since the second century $\mathrm{AD}$ in China $[14,15]$. To date, paper has been employed in lateral flow assays (LFAs), dipsticks and microfluidic paper-based analytical devices ( $\mu$ PAD) [16]. LFAs was first reported in 1956 by Plotz and Singer, who established the fundamentals of LFAs for latex agglutination assay [17]. Since then, the working principle of LFAs have been more elaborated and initiated extensive applications for rapid detection of infectious diseases [15]. LFAs have rapidly grown over the last few decades for qualitative and quantitative diagnosis and become a relatively mature technology [7, 18]. Currently, LFA market value is approximately 6.0 billion USD at a compound annual growth rate (CARG) of $7.7 \%$ and is expected to reach 8.7 billion USD by 2023 [19]. The LFA market is mainly driven by the high exposure to infectious disease throughout the world, aging population growth, high demand of POC testing and home-based LFA platform [19]. LFAs benefits from advantages such as long shelf lives - generally of 12-24 months without fridge, low cost, broad range 
of applications, small sample volume, and ease of use [7, 15]. Moreover, the sensitivity and specificity are determined by flow characteristics optimisation. It depends on membrane matrix and labels for biorecognition used in the assay to provide acceptable qualitative and semi-quantitative readout [7, $15]$.

During the last decade, several published review papers have discussed the recent applications of LFAs and novel materials for enhancing their performance $[2,7,20,21]$. The major trend in assay enhancement is developing novel materials to express more sensitive and specific to the target, or to have multiple functions [22-24]. In addition, sample preparation can be optimised and enhanced to control and prepare the samples before introducing them into the device [25, 26]. Current challenges must be clearly identified to better understand the limitations and to provide guidelines for assay performance improvement. Therefore, this review mainly focuses on challenges and provides perspectives to overcome them. The review also identifies future trends of employing advanced technologies in LFAs. A brief overview on materials, working principles as well as applications of conventional LFAs are also included to provide fundamentals for understanding LFAs and their design criteria.

\section{State of the art of LFAs}

\subsection{Materials for LFAs}

LFAs are portable strips assembled on a plastic backing card, consisting of different parts: sample pad, conjugate pad, flowing membrane and absorbent pad, as shown in Figure 1. The flowing membrane is the area performing the test, where the pre-immobilised reagents capture the analytes in the sample.

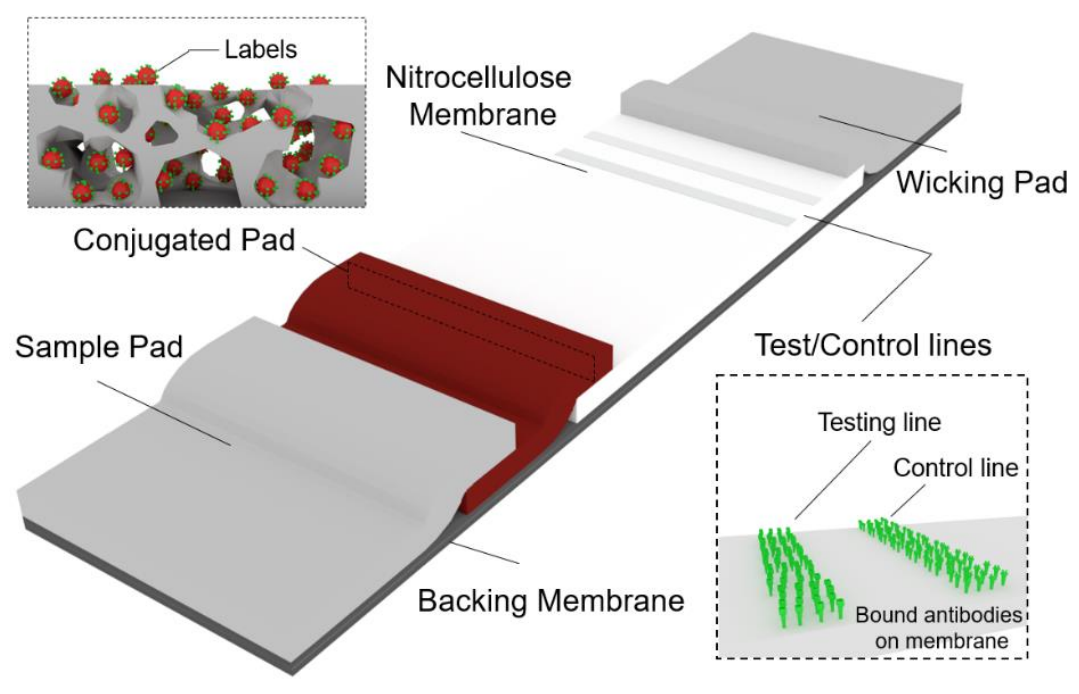

Figure 1. Conventional lateral flow assay. 
LFAs utilise a number of biorecognition molecules, labels and detection methods toward the diagnosis applications. The roles and material selection criteria are discussed below.

\subsubsection{Sample pad}

Sample pad is the first area to get in contact with the sample. The major purpose of a sample pad is to allow and maintain liquid sample to flow through continuously. In certain circumstances, if the concentration of the analytes in the sample is too high, filtration or dilution is required to treat the sample before passing it through the later parts [26]. Additionally, this part can be used to pretreat sample by depositing dry reagents to adjust $\mathrm{pH}$ for achieving proper conditions for an assay [15]. Therefore, the sample pad requires a high tensile strength when it is wet. If the pad fails, the sample cannot be delivered through the subsequent parts and the assay cannot be used for testing. The common materials for this section are cellulose, glass fiber, rayon or modified filtration matrices [7, $15,27]$.

\subsubsection{Conjugate pad}

In LFAs, each strip test needs a label for capturing analytes in a liquid sample and for generating a signal at the testing and control lines. The label is modified with protein linked passively or covalently for specific analytes existing in the liquid sample for biorecognition in this area. Substantial research on labels used for LFAs has been performed. Examples are nanoparticles made of gold [28-30], carbon [31, 32] and other metals [33] or composited metals [34, 35] as well as modified nanoparticles such as magnetic nanoparticles [36, 37], fluorescent nanoparticles [38-40] or quantum dot [22-24]. There are also alternative labels such as carbon nanotubes [41], enzymes [42] and liposome $[43,44]$. After passing through the sample pad, the analyte could be bound to the conjugated label, and then flow together. As a result, the most important characteristic of conjugate pad is releasing the label continuously as well as selecting and optimising labels for proper biorecognition [15]. Additionally, the matrix needs to provide low binding capacity to minimise naturally bond between the label and the matrix. The common materials used for conjugate pad are glass fiber, polyester or rayon. [7, 15, 27].

\subsubsection{Flowing membrane}

After following through the conjugate pad, the sample enters the membrane, where the testing and control area are bound with immobilised protein for capturing the analytes. This is the most significant part that determines the assay sensitivity. The porous characteristics of the membrane allow consistent flow of liquid sample, maintain protein stability bound to the membrane for the whole shelf life and prevent non-specific binding. In addition to the consistent flow in the membrane, the run time determined by the capillary flow rate of each membrane also affects the sensitivity of 
the assay because the speed of the fluid flow indicate the time exposure between the label and the capture probes at the testing and control areas $[15,27]$. The capillary flow rate depends on the physical properties of the membrane: pore size, pore size distribution and porosity [27]. Pore size is used to indicate the largest size of pore related to filter application, whereas pore size distribution determines the entire range of pore sizes in the membrane and defines the capillary flow rate [27]. Porosity is relevant to the liquid volume acquired by the membrane, so it impacts the distribution of reagents flowing in the membrane [27]. In addition to physical characteristics of the membrane, the properties of liquid flowing in the membrane also affect the capillary flow rate. Contact angle, viscosity and surface tension play important roles in regulating flow rate because the interaction between liquid and porous membrane is determined by the capillary force. The governing equations for fluid flow are discussed in the later section.

The stability of protein binding to the membrane mainly affect assay performance. Long-term bonding of protein prefers to employ the hydrophobic and hydrogen bonds [15]. Hence, selecting reagents for protein immobilisation needs careful consideration. Besides the flow and protein binding issues, non-specific binding is another concern to be considered. Non-specific binding can result in misinterpretation such as false positive result, which causes overstress to the patient $[15,27]$. The most common material for the flowing membrane is nitrocellulose because it is chemically modified with amine group which enhances protein immobilisation as well as the proper flow characteristics which can be controlled and provide acceptable reproducibility [7, 15, 27].

\subsubsection{Absorbent pad and backing materials}

After passing through the test and control areas, the sample get through to the absorbent pad. The purpose of this section is to continue wicking the liquid which allows the remaining labels and analytes to reach the test lines and prevents backflow which may cause false positive [26]. Consequently, the characteristic of this part is the ability to absorb liquid sample at the end of the device and high capacity for liquid drainage. The absorbent pad can improve assay sensitivity, because it ensures that all labels are wicked through the testing and control area. The same materials as the sample pad can be used for the absorbent pad [15, 27]. For backing materials, to improve the strength and ease of handling, all pads are generally backed with a plastic card. Moreover, the backing card can maintain the assay to provide consistency for the device during the operation. Otherwise, liquid sample may not flow properly in the paper-based device, leading to false results. Backing the assay can be done in two ways which are hot lamination and adhesive tape. The typical materials are polystyrene, polyester or adhesive polymer [7,27].

\subsection{Assay formats}


Detection of biorecognition molecules is categorised into two basic formats: sandwich assay and competitive assay, as shown in Figure 2. For sandwich assay, the analyte reacts with the label, and then form a label-analyte complex. Subsequently, the complex is captured at the test line by the interaction between the complex and capture probes, and then they aggregate to form the line. The free-analyte labels are also able to interact with capture probes at the control lines to confirm the validity of the assay using the same mechanism. For competitive assay, it is suitable for analytes, which are too small to bind the label. In the presence of analytes, they compete for binding site at the test line resulting in no aggregation to form at the testing line [45]. On the other hand, in the absence of analytes, there is no analyte competing at the testing line, so the result of the assay would show two lines [7, 21].

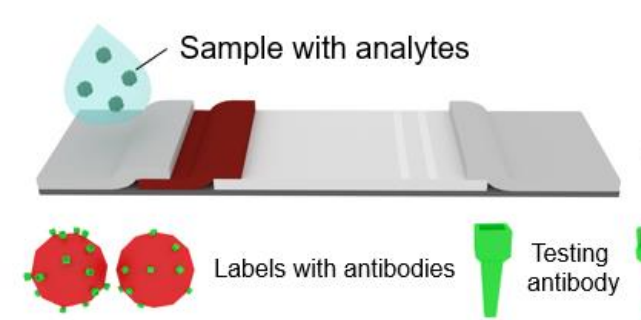

(a)

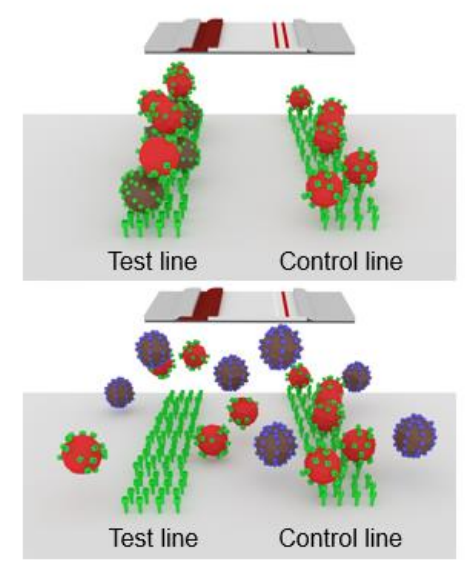

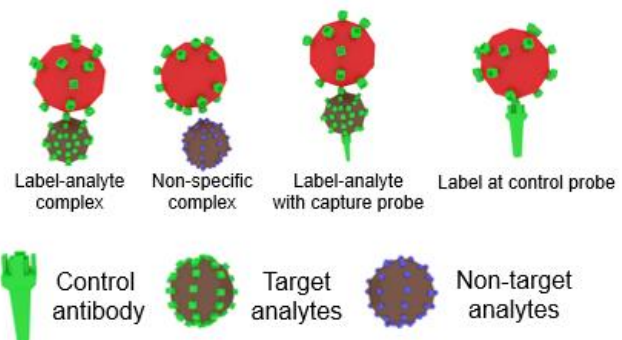

(b)

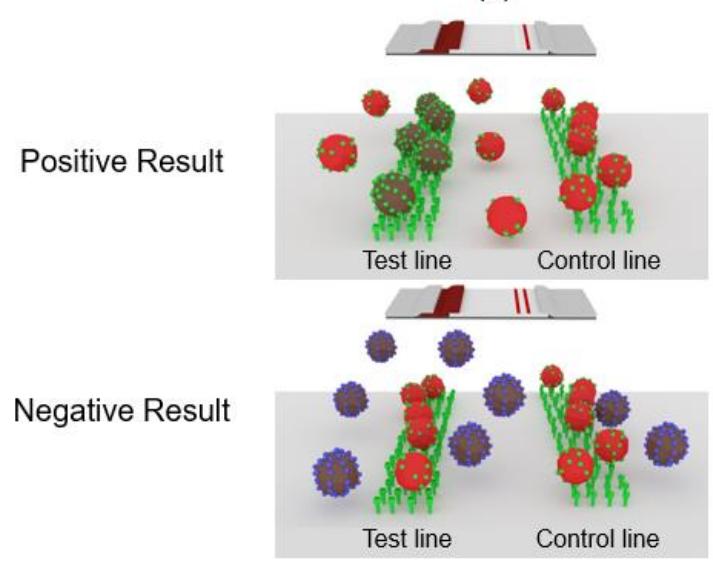

Figure 2. The mechanism of assay formats: (a) Sandwich assay; (b) Competitive assay.

\subsection{Working principle of LFAs}

We use the example of pregnancy strip test, to illustrate the working principle of LFAs. In an expectant mother, Human Chorionic Gonadotropin hormone (hCG) increases significantly in the body shortly after the fertilization and can be found in the urine after around $7-10$ days [46]. When the assay is dipped into the urine, the liquid is wicked through the sample pad and conjugate pad. In this step, if hCG exists in the urine, hCG will be bound to the colour particles conjugated with antibody-hCG and form a label-analyte complex. The complex flows together with the urine then pass through the test line that has another antibody-hCG which captures the complex with sandwich 
assay mechanism. Once the capture occurs, the complex aggregates and forms the signal at the testing line, determining that the owner of this urine is pregnant $[2,21,26]$. As the expression of hCG in an early expectant mother is significantly higher than a non-pregnant woman, the assay easily distinguishes these two groups. Nonetheless, in some diseases, the biomarker which is a disease indicator is extremely rare at the earlier stage. The sensitive assay is highly required to detect such a very low amount of the targets in the sample and get the treatment in time.

\section{Fundamental}

Flowing in porous media is mainly driven by capillary action resulting from hydrophilicity, surface tension and porosity defined by the media properties [2]. Capillary fluid transport can be categorised into two modes which are wet-out (dry) and fully-wetted conditions. In this section, the governing equations of flow conditions are discussed.

\subsection{Wet-out condition}

The fluid transport in wet-out condition of porous media can be explained by Washburn's equation considering the one-dimensional fluid flow through a bundle of small capillary tubes, neglecting gravity and evaporation. the equation of capillary filling is described as

$$
L^{2}=\frac{\gamma D t}{4 \mu} \cos \theta
$$

With the constant strip width, $L$ is the distance moved by fluid front $(\mathrm{m}), t$ is the flow time (s), $\gamma$ is the surface tension coefficient $(\mathrm{N} / \mathrm{m}), \cos \theta$ is the contact angle dependence, and $\mu$ is fluid viscosity (Pa.s). The fluid properties also determine the flow characteristic in the membrane. Therefore, sample treatment is preferred for some applications because of its impacts on the flow rate of the fluid in the membrane as well as on the sensitivity and the specificity of the assay $[7,26,27] . D$ is the average pore diameter $(\mathrm{m})$. It is worth noting that pore size reported from the supplier is the largest diameter for filtration application [27]. Ideally, the pore size can be measured by the largest hard particles able to pass through the membrane. However, this method is not preferred due to the complexity and impractical method to implement in QC process. Therefore, the pore size can be determined by other techniques such as bubble point, pressure required to push air through a wet membrane. As a result, the average pore diameter may introduce errors into the calculation. Equation (1) indicates that the distance moved by the fluid front is proportional to the square root of time [2, 47-50]. Consequently, various researchers investigated and added some neglected terms in Lucas-Washburn equation to the Newton's Second law to verify the derived solution and state the impact of neglected term [51-57]. Bosanquet et al. for instance added fluid inertia to the momentum balance equation and expressed the correlation of velocity term instead of acceleration toward a driving force [51]. 
Washburn's equation is used to predict the time of fluid transport in wet-out condition so that the strip developer can optimise parameters such as antibody concentration, label concentration and the length of the strip. This equation is limited to only to constant width strip. Fu et al. demonstrated that Washburn's relation is broken when the paper strip has an expanded or contracted section [58]. The flow in expansion or contraction part still maintained the linear relation between the fluid front distance and square root of time. However, the slope determined by the width of the channel was different. Therefore, this equation is used to approximately predict the time for fluid flow in the constant width. Generally, the supplier provides this information for customers to choose the membrane suitable for their applications.

\subsection{Fully-wetted condition}

When the fluid approaches the absorbent pad, the pad will absorb the remaining flow in the membrane due to the high density cellulose, which can acquire water and change the flow rate. Therefore, the fluid velocity is steady. The equation is derived initially from Navier-Stroke equation under the assumption of homogenization [59], reducing the equation described by Darcy's law in one dimension (1-D) as

$$
Q=\frac{-k A}{\mu l} \Delta P
$$

where $Q$ is the volumetric flow rate $\left(\mathrm{m}^{3} / \mathrm{s}\right), k$ is the permeability of the material to fluid $\left(\mathrm{m}^{2}\right), A$ is the area of the channel of the channel perpendicular to the flow $\left(\mathrm{m}^{2}\right), \Delta P$ is the pressure difference $(\mathrm{Pa})$ along the direction flow over the length $l(\mathrm{~m})$ [60]. Defining permeability coefficient of $k$ is still a problem in reality as the porous structure is not well-organized, because the paper is made of compressed cellulose fiber [2]. It is difficult to simulate a structure, which is random both in size and orientation [61]. Numerical simulation was employed for another option to solve this problem. The model can be obtained by simulating random models or using 3D imaging of the materials to generate a model for simulation. The common numerical method used to deal with complex and irregular shapes of porous media is lattice-Boltzmann method [61-64]. Alternatively, the porosity and permeability of the membranes was measured with empirical calculation by weighing the absorbed water volume to calculate velocity of fluid flow in different widths of the test strip [16].

Even though fully-wetted condition cannot be applied when the fluid firstly introduces to the paper strip due to the wet-out condition, Darcy's Law including its extended form of Brinkman model can also be used to understand the wicking mechanism and to improve assay performance [65]. Many studies applied Darcy-Brinkman model for velocity fluid in many configurations such as different sizes [16], wax-printed pillar [66] and PDMS barrier [67]. Nonetheless, the Darcy's Law cannot solve time-dependent problem because this equation is used for fully-wetted condition which means the 
paper strip needs to be first saturated, resulting in inaccurate prediction. Moreover, Darcy's law can be derived to simulate the electrical circuit analogy (ECA). The ECA model is a useful technique to explain wicking behaviour. The model utilise the analogy between fluid flow and electrical current. Briefly, the fluid flow rate, pressure drop, and fluidic resistance dominated by its geometry are analogous to current, voltage difference and electrical resistance respectively. The ECA provides a simple way to design a complex fluidic circuit. For instance, Toley et al. introduced the ECA model for designing the paper strip with shunt [68]. Similarly, some studies reported that the ECA model could be used and provided a good agreement with their experimental results [69-71]. Therefore, the ECA model can be used to explain the fluid flow of a wicking process with different paper strip width. However, this model required many parameters such as capillary pressure, porosity and permeability, which are experimentally determined and possibly result in variation in prediction.

\section{Fluid handling}

Conventional LFAs are known as a one-dimensional immunoassay. The flow rate can be dictated by the type of membrane including sample fluid properties. This section discusses potential applications for handling fluid flow in the assay including additional functions that can enhance the overall assay performance. This section categorised fluid handling into three main strategies: controlling the flow rate, mixing and separation.

\subsection{Controlling the flow rate}

In order to attain better and alternative ways to replace lab-bench approaches, a paper-based device requires a multistep process to allow chemicals to react in time sequences [72, 73]. Timing is a key in controlling and performing multistep assay on paper-based devices. Thereupon, understanding the flow and establishing a mathematical model to predict the liquid flow in the paper strip can be used to design and handle liquid sample in a predictable manner. There are a few methods for handling the fluid flow in paper-based devices, which are discussed as follow.

\subsubsection{Varying geometries}

Varying geometry of the paper strip can influence the fluid flow in the strip. Medina et al. studied the imbibition of different shapes of the paper strip by making use of Darcy's law and YoungLaplace equation as shown in Eq. (2) to predict the flow characteristic [74]. The team found that their theoretical prediction was in good agreement with the experimental data, and the model has the potential to predict more complex shapes. Mendez et al. used numerical simulation to predict and design the lateral flow device by varying shapes at the end of the paper strip without using the absorbent pad as shown in Figure 3a [75]. From the observation, fluid flow was divided into two phases. Fluid flow in the first phase was governed by Lucas-Washburn equation due to constant width 
of rectangular paper strip. However, when the fluid enters the second phase which was circularly expanded with different angles, the velocity of liquid front broke the Washburn's equation because of the sudden increase of dry bed space for the fluid to fill. The authors suggested that this configuration could be used to replace absorbent pad which wicked the excessive fluid in the system. The team found that experimental and computational data were in good agreement. Nevertheless, the computational data cannot be used for prediction in many cases. Every material used for LFAs has its variation due to the fabrication. Accordingly, the constant values and defined parameters used in the model are specific to a case and require validation before trying new materials. Furthermore, 1-D Darcy's law was applied to describe the flow in varying geometries by the ECA model. Fu et al. varied paper strip geometry using an equivalent system to predict and control the flow rate in the strip. For the expansion, from a small to a large width, the fluid velocity becomes slower because more time is needed to fill the pores in the wider segment. From this observation, the velocity of the liquid front was not obeying the Washburn's relation. Whereas, in the case of constriction, from a large to a small width, the fluid velocity remained the same [58]. The liquid front velocity still followed the Washburn's equation. Finally, according to the understanding of fluid flow in contraction-expansion paper model, a two-dimensional paper network (2DPN) depicted in Figure 3b was introduced by varying the geometry and had a potential to make a programmable paper network to achieve multistep process in a paper-based device $[76,77]$. Though the experiment result was in a good agreement with the computational result, Darcy's law may be limited in practice, which needs paper to be in a fully-wetted condition, resulting in error of the model.

(a)

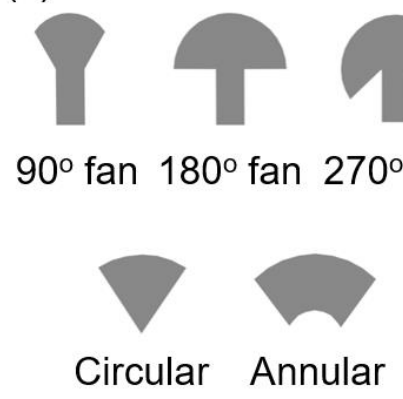

(b)

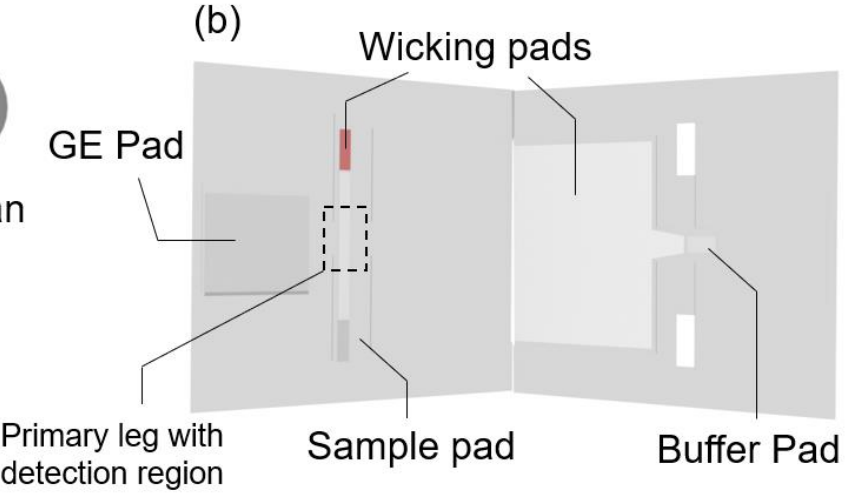

Figure 3. The schematic diagram to control flow rate: (a) The various geometries at the end of the paper strip without using absorbent pad [75]; (b) The use of different shapes to program the flow sequence [78].

\subsubsection{Fluidic valves}

The concept of valves introduces to control the fluid flow as an on/off switch. There are passive and active modes for controlling the fluid flow through a specific area. The passive method 
deposits the reagents or chemicals on the paper strip since the fabrication leads to delay of fluid transport. Various materials can be used to delay the fluid transport such as paraffin wax [79], surfactants [80] or dissolvable reagents such as sugar [78]. The concentration of reagents can indicate the delay of transport due to the solubility of the chemicals and fluid sample. However, the added chemicals may interfere with and reduce assay performance. Thus, using other chemicals as fluidic valve to block and delay fluids has to be carefully optimised. In addition, the physical delay was also reported by applying another cellulose pad on top of the main flow membrane called as a shunt as shown in Figure 4a, which flow can be delayed depending on size of the pad and the number of shunt used in the assay [68]. Additionally, the mechanical activated valve operated by the user was also reported. The foldable 2D paper in the cassette [76] and 3D foldable paper [72,81] shown in Figure $4 \mathrm{~b}$ also offer a simple way for the user to control the flow in the device.

On the other hand, active method requires automated external force is programmable for instance with electric $[82,83]$ and magnetic actuator [84, 85], Figure 4c. Regardless, using external power source and additional steps may reduce an ease of use and require more facilities, which are against the ASSURED guideline, so this issue needs to be taken into account in deciding the device concept.

(a)

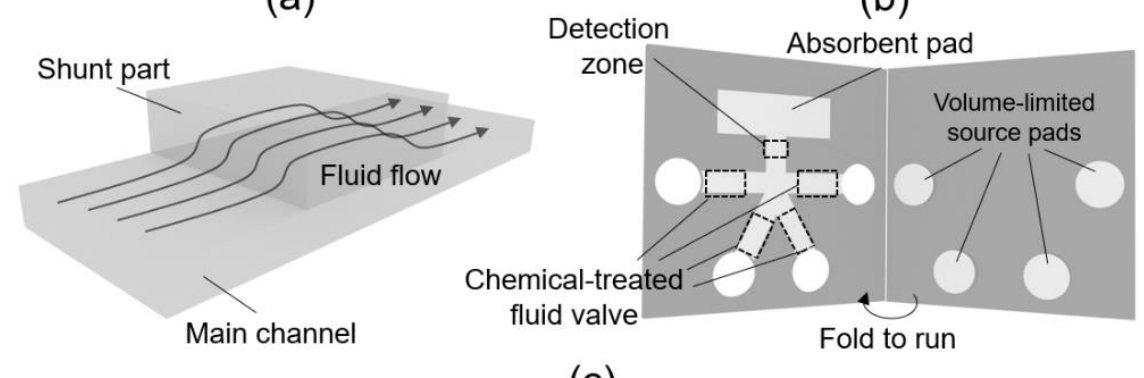

(c)

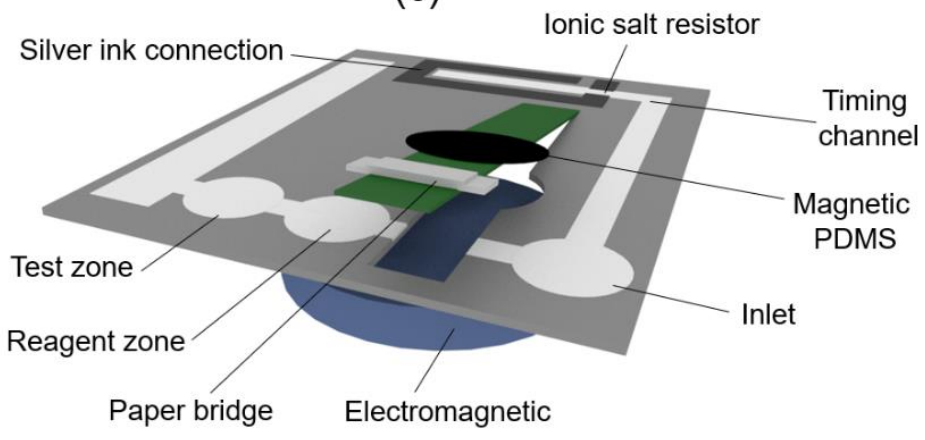

Figure 4. The schematic diagram for delaying flow in the channel: (a) the use of shunt to increase the flow distance [68]; (b) Chemical depositing to delay the flow [78]; (c) The magnetic actuator used to lift the channel to cut flow as a switch on/off valve [84].

\subsection{Mixing}


In addition to controlling fluid flow, mixing is another important fluid handling task in paper-based devices, particularly for processing reagents in the device. However, in contrast to conventional microfluidic devices, the irregular nature of the porous media leads to unpredictable and nonuniform flow through the paper [86]. Mixing in microscale relies on diffusion and chaotic advection due to the laminar flow in small geometries [87]. Therefore, increasing the contact surface between two different fluids and decreasing the diffusion path are required. Mixing can be categorised into passive and active methods. For passive mixing, the system does not require external forces to actuate mixing process, which is ideal for point-of-care diagnostic device [86]. Many studies demonstrated simple dye colour mixing in paper-based platform with different shapes of channel such as zigzag and curved channels altogether with various paper materials such as conventional Y-shaped Qualitative Grade 3 filter as well as polyester-cellulose paper shown in Figure 5a [86, 88]. Furthermore, a single sheet of paper was fabricated as three-dimensional channel by a unique photolithography method with two different masks [89]. This application illustrated the mixing by dispensing commercial dye and evaluated the mixing efficiency by colorimetric measurement. It is worth noting that transverse solute dispersion mainly dominated by pore space in porous network accomplished mixing in shorter distances than molecular diffusion. A foundation of transverse solute dispersion has been reported including theory and experiments in a recent study [90]. However, it may require an extensive study for implementing immunoassay strip application. On the other hand, an active method requires external force to generate disturbance for mixing such as electrically or acoustically induced forces. Surface acoustic wave (SAW) was used to perform mixing in paper-based device, Figure 5b. Single phase unidirectional transducer (SPUDT) electrodes deposited on crystal lithium niobate (NB) piezoelectric materials generated a Rayleigh SAW from SPUDT to focal point on the substrate, where the channel is located. Fast, uniform and consistent mixing were observed in the paper channel [86]. Moreover, the team also showed that SAW increased the flow rate of the fluid in the paper channel. Nevertheless, mixing in paper-based devices remains obscure. Precise and predictable model are required to better understand and design paper-based device for improved sensitivity and multistep assay [25].
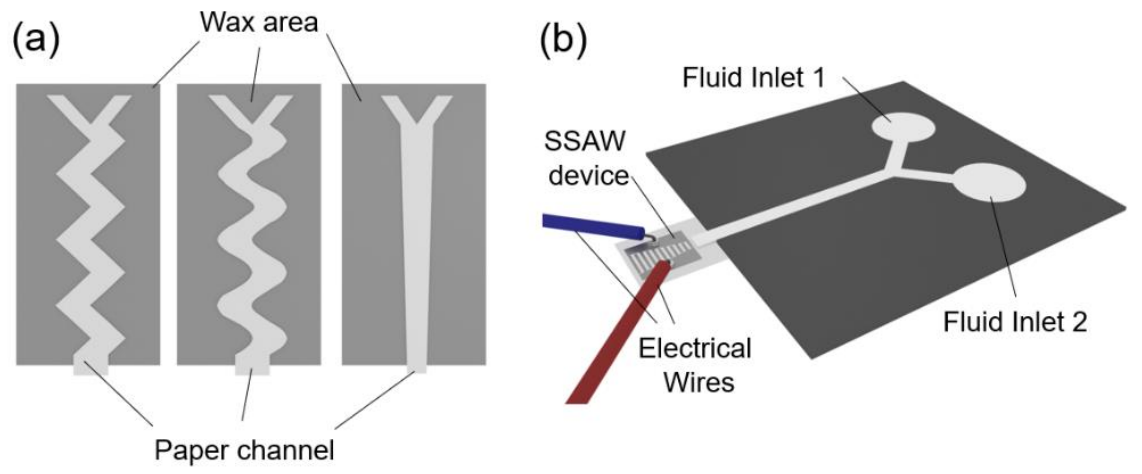
Figure 5. The schematic diagram for mixing in paper-based devices: (a) Passive method using various patterns such as zigzag, curved and straight Y-channel; (b) SAW activation for mixing enhancement [86].

\subsection{Separation}

Paper was introduced for separation applications since the 1940s, and known as paper chromatography $[91,92]$. This material was firstly used to separate amino acid sequence of insulin [93]. Since then, researchers have been using paper for various applications. In the 1950s, few studies involved protein characterization with electrophoresis. The protein stained the paper as a band depending on its molecular weight of each molecule under an electric field [94, 95]. This technique was refined and used as a conventional blotting, which is nowadays the gold standard for proteomic studies. Furthermore, separation on paper-based materials has been also extended to LFAs technology. Shiroma et al. demonstrated the use of chromatographic paper for electrochemical detection. Three integrated electrodes separate analytes with low voltage and can detect paracetamol and 4-aminophenol [96] as shown in Figure 6a. Zhong et al. used a simple geometry to separate free dye from the mixed protein solution and demonstrated the capability of controlling capillary action, which is potentially useful for future development of paper-based devices [88]. Due to the complex structure of the paper-based materials, it is difficult to control the interconnected pores for separation applications. Hence, further research is needed to better control the porous structure. Correspondingly, the porous paper must be optimised for applications with more complex solutions.

(a)

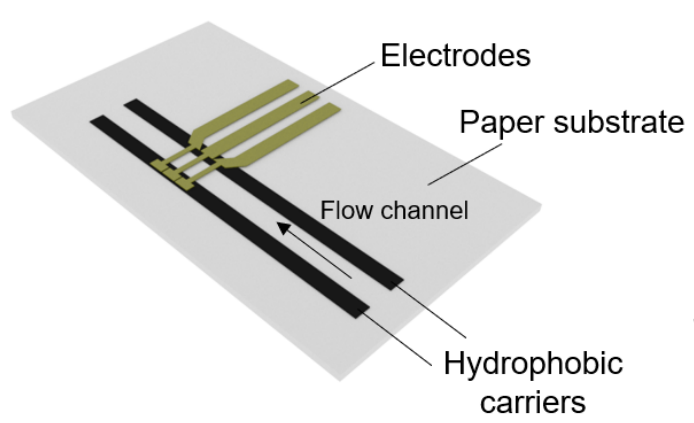

(b)

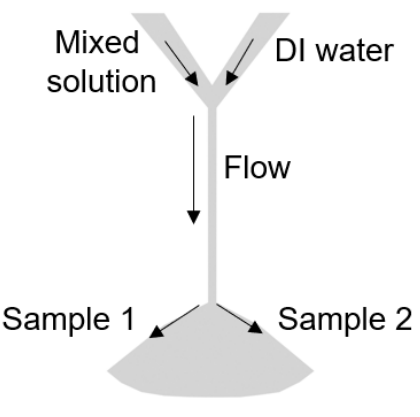

Figure 6. The schematic diagram for separation: (a) the use of paper-based electrochemical device to separate and detect paracetamol and 4-aminophenol [96]; (b) the illustration of protein separation using paper-based device for simple geometry [88].

\section{Current challenges}

Sensitivity and specificity are important indicators for validating LFAs. Conventional paperbased platform may not have enough sensitivity to perform well in clinical applications, otherwise 
the result can be misinterpreted as false negative and false positive, which will tremendously impact the patient through unsafe behaviour, unexpected consequent symptoms and mental problems such as anxiety or stress [97]. Therefore, the development of LFAs needs to overcome several current limitations, which are categorised into engineering and biochemical challenges, as summarised in Figure 7.

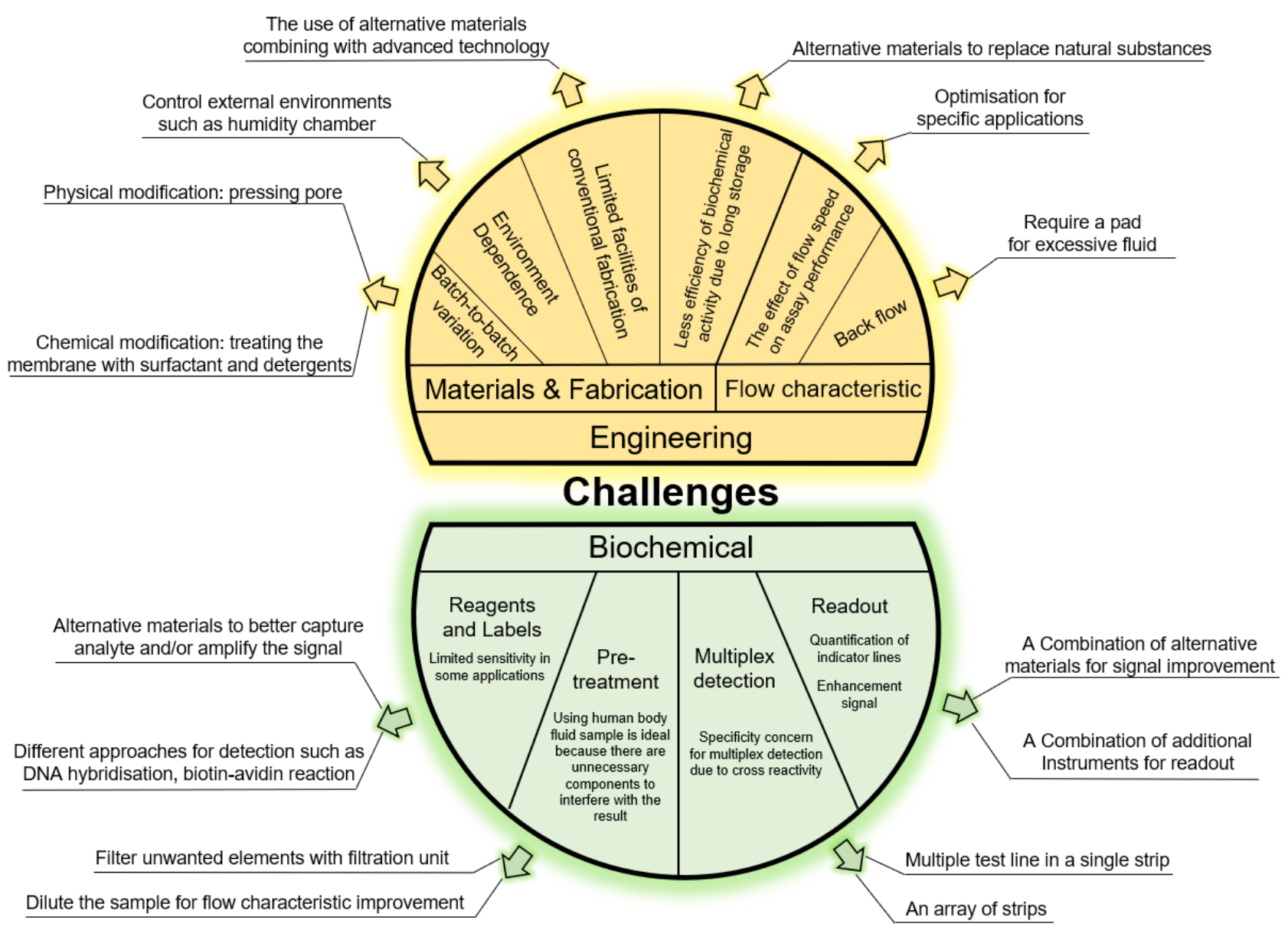

Figure 7. The summary diagram of the current challenges and possible solutions to improve the LFAs

\subsection{Engineering challenges}

\subsubsection{Materials and fabrication}

Paper-based materials lack control of physical properties such as capillary flow rate, surface area, pore size distribution, porosity, permeability, and wettability. Batch-to-batch variation leads to inconsistency particularly the difference in fabrication process from different manufacturers [98]. As a consequence, physical and chemical modification are required to treat the surface of paper-based devices to enhance fluid flow, colour uniformity and protein immobilisation [11]. For example, Park et al. pressed the nitrocellulose membrane to reduce the pore size causing delayed fluid flow [99]. Moreover, treating membrane with surfactant and detergents results in decreasing protein binding, but improving wettability of the membrane [27]. Excessive modification might also contaminate 
papers when it is exposed to other chemicals or reagents [11]. Therefore, manufacturers optimised the right detergent concentration to use with the membrane in the fabrication process. Furthermore, environmental factors such as humidity and temperature affect the quality of LFA products because nitrocellulose membrane is fragile, short shelf life and dependence on environment variation, resulting in invalid evaluation of strip test [21]. Controlling the storage and test conditions is another approach for improving the reproducibility of LFAs. However, it is impractical for use in a harsh environment such as a field test.

Devising alternative materials is still challenging as novel materials need to prove its performance and gain acceptance from the market. Further risk is from the cost of manufacturing development and scaling up for mass production. Some researchers suggested that the development of new materials and improved facilities or technology are preferred to better control the LFA performance [15]. Advanced printing technology which is one of promising technology can deposit a broad range of materials such as metals and nanoparticles might improve the assay performance and yield better reproducibility [100]. For example, Yu et al. demonstrated inkjet-printing surfaceenhanced Raman spectroscopy (SERS) on paper-based surface swab and lateral-flow dipstick for trace chemical detection [101]. The paper absorbed the chemical and was later analysed with SERS. Moreover, Enzymatic solution such as horseradish peroxidase (HRP) could be printed with piezoelectric inkjet printing without damaging its properties [102, 103]. Even though many biomolecules could be printed, the viscosity of the solution and biological properties are a major concern requiring considerable optimisation.

In addition to paper engineering, the selection of reagents in LFAs also impacts their reproducibility. The simple immobilisation of reagents is dispensing or dipping and drying in a controlled condition. The dissolution of reagents is based on inherent solubility, particle size, viscosity, flow rate, $\mathrm{pH}$, ionic strength of the solvent, porosity, uniformity of paper matrix [104, 105]. Likewise, another challenge involving chemicals used in the LFAs is preserving biochemical activity in the strip such as sugars (discussed later in the section 5.2.4 Readout) [106-108]. The ideal LFAs need a long shelf live without refrigeration as stated by the WHO guideline. On the contrary, some assays using natural reagents requires special care to preserve the biomolecules, leading to invalid and inefficient testing in remote locations or in a harsh environment. As a result, many researchers attempted to replace natural biomolecules by developing aptamers, artificial receptors which is specific to the target, or nanozymes, nanoparticles possessing enzyme-like activity $[100,109,110]$. Recently, these units were successfully proved the functionality of rehydration and biorecognition in LFAs for the possibility to replace natural reagents in LFAs [111, 112]. Therefore, market-ready LFAs need to be carefully optimised in terms of physical properties and reagents to ensure that they 
do not suffer from reproducibility and technical issues originating from the fabrication process and the materials.

\subsubsection{Flow characteristics}

Capillary flow rate is influenced by the different substrates provided by the manufacturers. For example, Millipore provides broad options of Hi-Flow Plus nitrocellulose membrane from HF075 to HF240. The number indicated the required time in seconds for the fluid to reach a distance of 4 $\mathrm{cm}$. It is worth noting that the membrane with faster capillary flow rate has a higher specificity and lower sensitivity [27]. For instance, Mao et al. demonstrated that they can significantly get a higher signal at the test line with HF240 rather than with HF180 [113]. Any assay requires the optimal point among the parameters: sample working volume, sensitivity, specificity and especially assay reaction times. Furthermore, the backflow in the absence of absorbent pad or defect in the paper might occur, leading to false positive signal. Thus, the completeness and robustness of each LFA device also need to be concerned.

\subsection{Biochemical challenges}

\subsubsection{Reagents and labels}

One of the most important components determining the sensitivity and specificity is the label for capturing target analytes in the liquid sample. The most common material used in LFAs is colloidal gold nanoparticles due to the ease of functionalization for better specificity, chemical inertness and its exceptional optical properties [114]. However, this material is limited for getting a higher sensitivity in some clinical applications. The diagnosis of some diseases may require additional steps to purify the sample for detecting rare target analytes. The additional steps are against the concept of equipment-free POC and difficult to penetrate the clinical practice. Consequently, various research groups have introduced alternative labels as mentioned in the previous section to better capture the target analytes and amplify the signal leading to improving sensitivity and specificity [20]. Furthermore, the selecting type of biorecognition needs to be carefully considered. Typically, conventional LFAs employed immunoassays, which relies on binding between specific antigen and antibody. To date, many studies have been reporting different approaches such as nucleic acid hybridization or avidin-biotin reaction $[21,26,71,113,115,116]$. Thus, biorecognition activities among these biomolecules is another critical challenge to be addressed for enhancing the sensitivity and specificity of the assay.

\subsubsection{Pre-treatment challenges}


Using human body fluid sample such as blood, urine and saliva without a preparation step is ideal for performing LFAs in clinical applications [7]. Complex compound suspending in blood sample or others impacts assay performance, so it is recommended to filter unnecessary elements out before loading into the LFAs [7]. These elements can chemically block the biorecognition events at the conjugate pad or testing line leading to false negative. In the case of blood, complex biomolecules and the high viscosity also affect the flow in the membrane and the sensitivity of the assay [27]. In some cases, a high concentration of analytes in the liquid sample also results in false negative, which is known as the Hook effect [117]. Many solutions also have been provided without using lab-bench equipment such as simple dilution with buffer or filter cassette for red blood cells separation [26]. Therefore, LFA developers have to design additional components, if sample preparation is needed for easy handling.

\subsubsection{Multiplex detection}

In a common practice, several analytes are required to be detected for the accurate diagnosis and confirmation of the disease in one patient. A multiplex assay would be ideal for performing multiple analytes detection in a single test. There are a few assays reported to date. For example, Dineva et al. deposited different colour particles and multiple capture probes with different test lines. The assay provided the semi-quantitative result by evaluating the colour intensity with score [118]. This strategy was implemented for various diseases with different biomarkers in individual test strips [119-122]. Different methods such as multiple parallel channel, star-shape, or arrays of strip were also reported [123-126]. The major concern of multiplex detection is the specificity of the multiplex assay due to cross reactivity particularly when the assay is involved with multiple analytes in the liquid sample. Therefore, the selection of immunological probes in the testing strip needs to be carefully optimised.

\subsubsection{Readout}

Conventional LFAs are considered as a qualitative or semi-quantitative assay. The assay only requires some threshold to highlight the indicator line by particle aggregation after binding specifically between label-analyte complex and capture probe known as immunoassay. However, a quantitative assay needs a tool to evaluate the signal such as colour intensity or electrochemical signal, which requires multi-function labels to produce a clearer or more amplified signal to be better quantified by the reader. For a colorimetric assay, evaluating by naked eyes results in human bias and misinterpretation [106]. Thus, the assay needs a reader to digitize the data from colour to numerical data. The advancement in smartphone technology allows images to be taken and analysed immediately $[13,100,127]$. Nonetheless, image quality may vary depending on different software 
and hardware as well as environmental factors such as the amount of light while taking the image [106]. Thereupon, self-calibration strategies were employed to solve the image quality issue [128130]. Moreover, the development of smartphone-based paper sensor rapidly increases in a last few years [131]. Image acquisition, high quality of camera and image processing application are combined to analyse the data, which are successfully demonstrated in a broad range of applications such as monitoring environmental pollutions $[132,133]$ or disease screening $[134,135]$.

Furthermore, stability of the reagents used in the assay also affect the outcome signal [1]. Some studies have made use of enzymatic activity to perform colorimetric assay. However, improper storage can cause loss of activity including external environments such as temperature, $\mathrm{pH}$ and humidity [106, 108]. Additives such as sugars are used to stabilise enzyme in the assay [106, 107]. For example, Trehalose was firstly used with enzymatic assay [108]. Trehalose can keep a large amount of water, which helps to maintain the enzyme fresh and active during storage period. Other sugars like dextran are also implemented for the same purposes. Thus, the reagent preservation is one of the potential strategies to allow the signal reader to better analyse the data.

In addition, several research groups have made use of luminescent labels such as fluorescent, chemiluminescent or electro-chemiluminescent detection. Luminescent signal can reduce the limit of detection less than that of colorimetric assays by reducing background noise, which is considered as more sensitive and quantitative detection methods [136-138]. However, luminescent assays require a reader with specific excitation and emission filters. Hence, the development of the reader and label selection plays an important role in the measurement and quantification of signal intensity [11] In summary, detecting biological targets such as proteins circulating in bloods may require quantitative data for clinical practice, as these data are important for screening the patient for preliminary diagnosis or rapidly acquiring the data for follow-up results.

\section{Conclusions and Perspectives}

LFA technology has evolved for over 50 years. Equipped with the ease of use, affordability and rapid diagnosis, LFAs have been employed in a broad range of applications from health monitoring, veterinary field work, environmental testing, agricultural goods and other industries. Being a mature technology, LFAs have been proven overtime for maintaining the quality of the assay, which are sensitivity, specificity, reproducibility and assay stability, for mass production and market acceptance. A huge change in manufacturing process or technological disruption would lead to high cost of investment [15]. Thus, further development of LFAs would need a novel detection concept offering more sensitivity and specificity without much changing in fabrication process and more controllable system to better enhance reproducibility to maximise the assay performance. On the other 
hand, recent paper-based platforms such as $\mu \mathrm{PAD}$ having initiated for a few decades ago have attracted considerable attention from researchers and developers of POC diagnosis. The platform technology also requires reproducible fabrication process to maintain the assay performance and reliable diagnosis. The development of diagnostic assays also needs venture capitalist to support financially to further improve the assay performance or to establish trustworthy and controllable facilities. Furthermore, due to the complex structure of cellulose-based materials resulting in poor reproducibility, it is difficult to predict and simulate flow in the porous membrane with analytical or numerical models. Understanding the physics of paper-based system is one of the possibilities to better control the flow in the porous membrane and open up the technology to more applications.

Early-stage disease detection is vital for medical diagnosis. The earlier the disease is detected; the higher is the survival rate of the patient because the treatment can start in time. In some cases, the survival rate of early-detected cancer can reach up to 93\% [139]. However, at an earlier stage, a disease does not show any symptom and produces a minute amount of specified biological markers, which is very difficult to detect in common LFAs. Therefore, developing a sensitive and specific LFAs to detect rare cells is required. Several ways to develop assay performance are discussed here. Improving materials for multifunctionalities to avoid sample pretreatment is implemented [7]. For instance, an additional filtration cassette can be utilised to remove unnecessary molecules out within minutes before loading the sample into the lateral flow device as shown in Figure 8a [26]. Selffiltration function could enhance sensitivity and specificity because some elements which interfere with the detection process are already removed. In addition, adapting some parts in LFAs was also demonstrated. Many studies combined additional conjugated pads which are specific to nanoparticles in the other conjugated pad as shown in Figure $8 \mathrm{~b}[140,141]$ to amplify the signal as a testing line. Moreover, a multiplex platform is required to confirm the existence of the disease, because identifying some diseases requires multiple biomarkers for the diagnosis especially the detection of rare cells. Thus, detecting multiple analytes with a single test would be ideal as shown in Figure 8c. There have been several studies performing multiple lines to detect targets in the sample, which is probably used to detect multiple rare analytes $[142,143]$. Correspondingly, combining with advanced material technology, novel materials and new detection techniques can be used to perform in LFAs for signal enhancement and amplification to better detect rare cells in human samples. The label may perform as a dual-function sensor which are detecting rare cells in human sample directly before purification and reacting with specific chemicals or external stimulus to emit the signal at a testing area. All possible strategies published recently require an extensive study and knowledge transfer from the lab-bench protocol to commercial products launched in the market. 
(a)

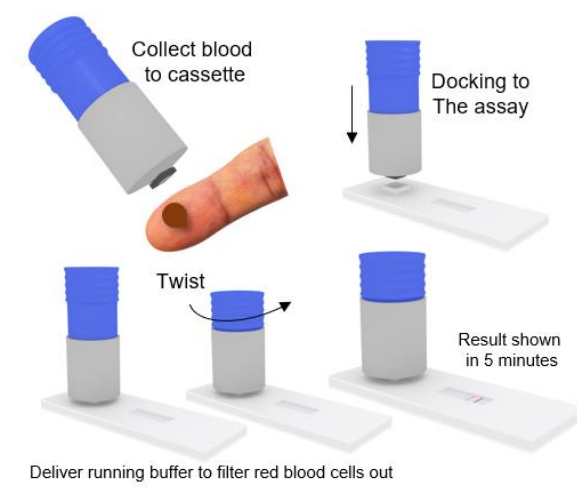

(b)

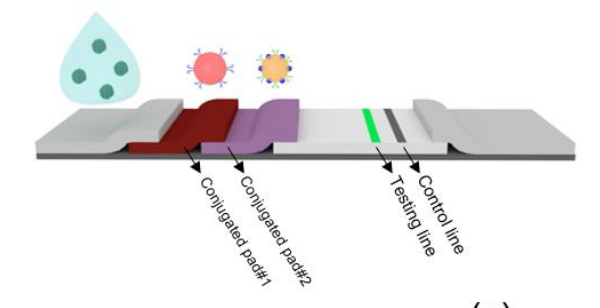

(c)

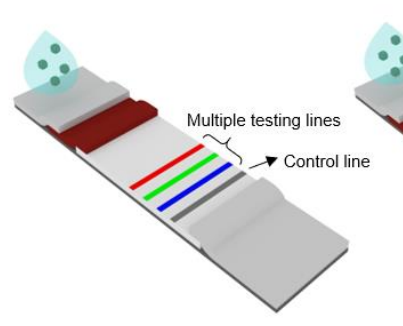

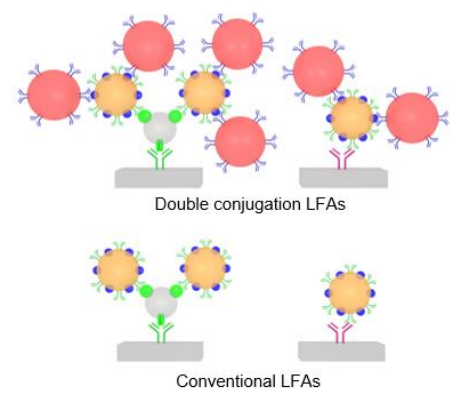

Conventional LFAs

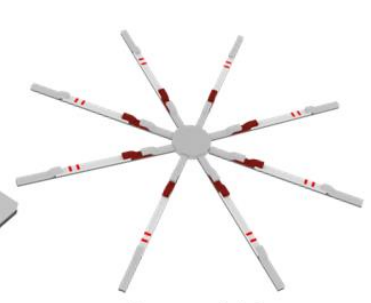

An array of strips

Figure 8. Possible strategies for improving assay performance: (a) The cassette for collecting blood, filtering red blood cells out, delivering the running buffer [26]; (b) Double conjugation by adding second conjugated pad to amplify the signal [140]; (c) Multiple testing lines, parallel channels in a single strip and an array of strip test capturing different targets in the samples [123, 124, 126].

The major limitation is the fact that LFAs are qualitative or semi-quantitative assays. Typically, the signal generated in the LFAs is the colour intensity that only reports yes/no result. Most clinical applications however require quantitative data of proteins, nucleic acids and other markers [21]. Many research groups have offered all-in-one devices to collect, analyse and quantify the data in one device. Recently, there are several paper-based devices with integrated circuits and selfpowered systems have been demonstrated for monitoring glucose [144-146]. Cho et al. demonstrated paper-based sensor patch with a self-powered system. Sweat was wicked through the direction shown in Figure 9a. When the sweat reaches the hydrophilic reservoir layer, the glucose in the sweat reacts with glucose oxidase (GOx) on the paper and generate free electron by oxidation reaction. In this design, the device can be performed without external power source because it made use of enzymatic fuel cells [147-149] which GOx layer (Anode) oxidized glucose and air cathode (Cathode) reduce oxygen. Thus, the current output as a transducing signal was related to the glucose concentration [145]. Xiao et al. reported the use of paper-based patch for monitoring glucose, Figure 9b. The patch was equipped with wearable clothes. Sweat from the volunteer during exercise is wicked sweat by capillary forces from absorbent pad to testing area where the glucose oxidase and colorimetric assay were deposited. The result was analysed with colorimetric assay by a smartphone [146]. However, the amount of sweat generated by the users, type of exercises and environment conditions such as temperature or humidity may result in a variation of tests. To overcome these issues, advanced technologies of smartphones or self-powered system and artificial intelligence (AI) enable on-site 
testing to become easier due to its multifunction, such as taking high quality photos, analysing data with AI or uploading data for cloud computing. With the onset of advanced technology, it also opens up another medical diagnostic trend such as the shift in healthcare to a more personalised approach, leading to home-based health monitoring and on-site testing [100].

With the emerging of supportive data infrastructure such as $5 \mathrm{G}$ technology, cloud data storage and retrieval, telemedicine will be possible $[2,150]$. In the foreseeable future, people can access the assay at the pharmacy near their home with an affordable price. They can do the test at home within minutes, and then upload the data within seconds directly and confidentially to the doctor. Accordingly, patients can ask for a private consultation with the practitioners using video calls. If the test result is abnormal, the doctor can generate electronic prescription. In more serious cases, the doctor is able to call for further diagnosis if the result potentially develops an early stage of some severe diseases such as cancer. All steps can be done without going to the hospital, thus reducing cost, time and risk of spreading the disease in the public. In conclusion, the combination of LFAs and paper-based platforms as well as smartphone is a trend toward better qualitative and quantitative analyses, offering an alternative for preliminarily disease screening at home, on-site as well as in remote locations without the need for lab-bench equipment. 


\section{(a)}
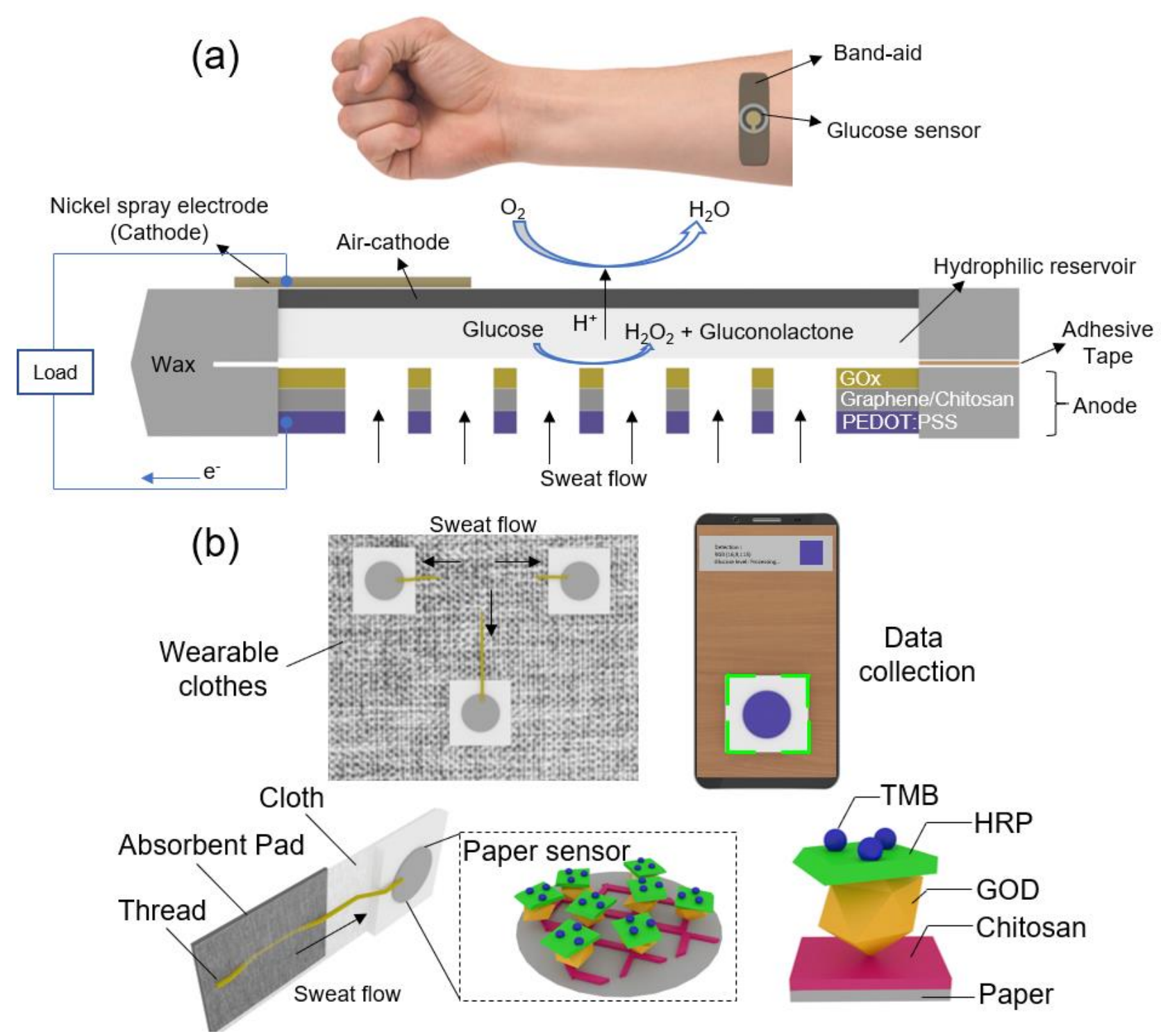

Figure 9. The use of paper-based sensor to monitor glucose: (a) Combining with a self-powered system on band-aid tape with current response from the electrochemical activity [145]; (b) Attaching with wearable clothes to monitor glucose with a colorimetric assay with smartphone [146].

\section{Acknowledgements}

The authors acknowledge the support of the Australian Research Council (DP180100055) and higher degree research scholarships GUIPRS and GUPRS Scholarships to S.K. from the Griffith University. 


\section{Reference}

[1] G. G. Morbioli, T. Mazzu-Nascimento, A. M. Stockton, and E. Carrilho, "Technical aspects and challenges of colorimetric detection with microfluidic paper-based analytical devices ( $\mu$ PADs) - A review," Analytica Chimica Acta, vol. 970, pp. 1-22, 2017/06/01/ 2017.

[2] A. K. Yetisen, M. S. Akram, and C. R. Lowe, "Paper-based microfluidic point-of-care diagnostic devices," Lab on a Chip, 10.1039/C3LC50169H vol. 13, no. 12, pp. 2210-2251, 2013.

[3] P. Yager, G. J. Domingo, and J. Gerdes, "Point-of-Care Diagnostics for Global Health," Annual Review of Biomedical Engineering, vol. 10, no. 1, pp. 107-144, 2008/08/01 2008.

[4] K. D. Clark, C. Zhang, and J. L. Anderson, "Sample Preparation for Bioanalytical and Pharmaceutical Analysis," Anal Chem, vol. 88, no. 23, pp. 11262-11270, 2016/12/06 2016.

[5] S. Kasetsirikul, J. Buranapong, W. Srituravanich, M. Kaewthamasorn, and A. Pimpin, "The development of malaria diagnostic techniques: a review of the approaches with focus on dielectrophoretic and magnetophoretic methods," (in eng), Malar J, vol. 15, no. 1, pp. 358-358, 2016.

[6] G. Millot, B. Voisin, C. Loiez, F. Wallet, and S. Nseir, "The next generation of rapid point-of-care testing identification tools for ventilator-associated pneumonia," (in eng), Ann Transl Med, vol. 5, no. 22, pp. 451-451, 2017.

[7] M. Sajid, A.-N. Kawde, and M. Daud, "Designs, formats and applications of lateral flow assay: A literature review," Journal of Saudi Chemical Society, vol. 19, no. 6, pp. 689-705, 2015/11/01/ 2015.

[8] P. K. Drain et al., "Diagnostic point-of-care tests in resource-limited settings," (in eng), Lancet Infect Dis, vol. 14, no. 3, pp. 239-249, 2014.

[9] W. Grace and M. H. Zaman, "Low-cost tools for diagnostic and monitoring HIV infection in lowresource settings," Bulletin of the World Health Organization, vol. 90, no. 12, pp. 914-920, 2012.

[10] P. T. Mashamba-Thompson, A. N. Jama, B. Sartorius, K. P. Drain, and M. R. Thompson, "Implementation of Point-of-Care Diagnostics in Rural Primary Healthcare Clinics in South Africa: Perspectives of Key Stakeholders," Diagnostics, vol. 7, no. 1, 2017.

[11] D. M. Cate, J. A. Adkins, J. Mettakoonpitak, and C. S. Henry, "Recent Developments in Paper-Based Microfluidic Devices," Anal Chem, vol. 87, no. 1, pp. 19-41, 2015/01/06 2015.

[12] X. Jiang and Z. H. Fan, "Fabrication and Operation of Paper-Based Analytical Devices," Annual Review of Analytical Chemistry, vol. 9, no. 1, pp. 203-222, 2016/06/12 2016.

[13] S. Hosseini, P. Vázquez-Villegas, and S. O. Martínez-Chapa, "Paper and Fiber-Based Bio-Diagnostic Platforms: Current Challenges and Future Needs," Applied Sciences, vol. 7, no. 8, 2017.

[14] Rooz. (2010, 02,07). The power of Paper: Elegant Solutions in Diagnostics [Online]. Available: https://miter.mit.edu/articlepower-paper-elegant-solutions-diagnostics/.

[15] R. Wong and H. Tse, Lateral Flow Immunoassay, 1 ed. Humana Press, 2009, p. 224.

[16] C. Parolo, M. Medina-Sánchez, A. de la Escosura-Muñiz, and A. Merkoçi, "Simple paper architecture modifications lead to enhanced sensitivity in nanoparticle based lateral flow immunoassays," Lab on a Chip, 10.1039/C2LC41144J vol. 13, no. 3, pp. 386-390, 2013.

[17] J. M. Singer and C. M. Plotz, "The latex fixation test: I. Application to the serologic diagnosis of rheumatoid arthritis," The American Journal of Medicine, vol. 21, no. 6, pp. 888-892, 1956/12/01/ 1956.

[18] P. Yager et al., "Microfluidic diagnostic technologies for global public health," Nature, vol. 442, no. 7101, pp. 412-418, 2006/07/01 2006.

[19] M. R. Report, "Lateral Flow Assay Market by Application, Product, Technique, End User - Global Forecast to 2023," Nov 2018 2018, [Online]. Available:

https://www.marketsandmarkets.com/Market-Reports/lateral-flow-assay-market167205133.html, Accessed on: 3 July 2019.

[20] E. B. Bahadır and M. K. Sezgintürk, "Lateral flow assays: Principles, designs and labels," TrAC Trends in Analytical Chemistry, vol. 82, pp. 286-306, 2016/09/01/ 2016. 
[21] J. Hu et al., "Advances in paper-based point-of-care diagnostics," Biosensors and Bioelectronics, vol. 54, pp. 585-597, 2014/04/15/ 2014.

[22] X. Chen et al., "Development of a rapid and sensitive quantum dot-based immunochromatographic strip by double labeling PCR products for detection of Staphylococcus aureus in food," Food Control, vol. 46, pp. 225-232, 2014/12/01/ 2014.

[23] X. Li et al., "A fast and sensitive immunoassay of avian influenza virus based on label-free quantum dot probe and lateral flow test strip," Talanta, vol. 100, pp. 1-6, 2012/10/15/ 2012.

[24] J. Shen et al., "Immunochromatographic assay for quantitative and sensitive detection of hepatitis B virus surface antigen using highly luminescent quantum dot-beads," Talanta, vol. 142, pp. 145$149,2015 / 09 / 01 / 2015$.

[25] C. Carrell et al., "Beyond the lateral flow assay: A review of paper-based microfluidics," Microelectronic Engineering, vol. 206, pp. 45-54, 2019/02/01/ 2019.

[26] B. O'Farrell, "Lateral Flow Technology for Field-Based Applications-Basics and Advanced Developments," Topics in Companion Animal Medicine, vol. 30, no. 4, pp. 139-147, 2015/12/01/ 2015.

[27] Rapid Lateral Flow Test Strip: Considerations for Product Development, Millipore Corporation, 2013.

[28] W. Jawaid et al., "Development and validation of the first high performance-lateral flow immunoassay (HP-LFIA) for the rapid screening of domoic acid from shellfish extracts," Talanta, vol. 116, pp. 663-669, 2013/11/15/ 2013.

[29] J.-H. Lee et al., "Multiplex diagnosis of viral infectious diseases (AIDS, hepatitis C, and hepatitis A) based on point of care lateral flow assay using engineered proteinticles," Biosensors and Bioelectronics, vol. 69, pp. 213-225, 2015/07/15/ 2015.

[30] P. Mdluli et al., "Gold nanoparticle based Tuberculosis immunochromatographic assay: The quantitative ESE Quanti analysis of the intensity of test and control lines," Biosensors and Bioelectronics, vol. 54, pp. 1-6, 2014/04/15/ 2014.

[31] J. Bogdanovic et al., "Rapid detection of fungal $\alpha$-amylase in the work environment with a lateral flow immunoassay," Journal of Allergy and Clinical Immunology, vol. 118, no. 5, pp. 1157-1163, 2006/11/01/ 2006.

[32] M. O'Keeffe et al., "Preliminary evaluation of a lateral flow immunoassay device for screening urine samples for the presence of sulphamethazine," Journal of Immunological Methods, vol. 278, no. 1, pp. 117-126, 2003/07/01/2003.

[33] J.-M. Park, H.-W. Jung, Y. W. Chang, H.-S. Kim, M.-J. Kang, and J.-C. Pyun, "Chemiluminescence lateral flow immunoassay based on Pt nanoparticle with peroxidase activity," Analytica Chimica Acta, vol. 853, pp. 360-367, 2015/01/01/ 2015.

[34] T. Jiang et al., "Sensitive detection of Escherichia coli O157:H7 using Pt-Au bimetal nanoparticles with peroxidase-like amplification," Biosensors and Bioelectronics, vol. 77, pp. 687-694, 2016/03/15/ 2016.

[35] J. Yan et al., "Effect of physiochemical property of Fe304 particle on magnetic lateral flow immunochromatographic assay," Sensors and Actuators B: Chemical, vol. 197, pp. 129-136, 2014/07/05/ 2014.

[36] D. Duan et al., "Nanozyme-strip for rapid local diagnosis of Ebola," Biosensors and Bioelectronics, vol. 74, pp. 134-141, 2015/12/15/ 2015.

[37] Y. Wang, H. Xu, M. Wei, H. Gu, Q. Xu, and W. Zhu, "Study of superparamagnetic nanoparticles as labels in the quantitative lateral flow immunoassay," Materials Science and Engineering: $C$, vol. 29, no. 3, pp. 714-718, 2009/04/30/ 2009.

[38] L. Li et al., "Development of up-converting phosphor technology-based lateral-flow assay for rapidly quantitative detection of hepatitis B surface antibody," Diagnostic Microbiology and Infectious Disease, vol. 63, no. 2, pp. 165-172, 2009/02/01/ 2009.

[39] T. Salminen, E. Juntunen, N. Khanna, K. Pettersson, and S. M. Talha, "Anti-HCV immunoassays based on a multiepitope antigen and fluorescent lanthanide chelate reporters," Journal of Virological Methods, vol. 228, pp. 67-73, 2016/02/01/ 2016. 
[40] E. Juntunen, T. Myyryläinen, T. Salminen, T. Soukka, and K. Pettersson, "Performance of fluorescent europium(III) nanoparticles and colloidal gold reporters in lateral flow bioaffinity assay," Analytical Biochemistry, vol. 428, no. 1, pp. 31-38, 2012/09/01/ 2012.

[41] W. Qiu et al., "Carbon nanotube-based lateral flow biosensor for sensitive and rapid detection of DNA sequence," Biosensors and Bioelectronics, vol. 64, pp. 367-372, 2015/02/15/ 2015.

[42] K.-K. Fung, C. P.-Y. Chan, and R. Renneberg, "Development of enzyme-based bar code-style lateralflow assay for hydrogen peroxide determination," Analytica Chimica Acta, vol. 634, no. 1, pp. 8995, 2009/02/16/ 2009.

[43] K. A. Edwards and A. J. Baeumner, "Optimization of DNA-tagged dye-encapsulating liposomes for lateral-flow assays based on sandwich hybridization," Analytical and Bioanalytical Chemistry, vol. 386, no. 5, pp. 1335-1343, 2006/11/01 2006.

[44] H.-W. Wen, W. Borejsza-Wysocki, T. R. DeCory, and R. A. Durst, "Development of a competitive liposome-based lateral flow assay for the rapid detection of the allergenic peanut protein Ara h1," Analytical and Bioanalytical Chemistry, vol. 382, no. 5, pp. 1217-1226, 2005/07/01 2005.

[45] C. Liu et al., "Lateral Flow Immunochromatographic Assay for Sensitive Pesticide Detection by Using Fe3O4 Nanoparticle Aggregates as Color Reagents," Anal Chem, vol. 83, no. 17, pp. 6778-6784, 2011/09/01 2011.

[46] L. Wide, "Early Diagnosis of Pregnancy," The Lancet, vol. 294, no. 7626, pp. 863-864, 1969/10/25/ 1969.

[47] J. M. Bell and F. K. Cameron, "The Flow of Liquids through Capillary Spaces," The Journal of Physical Chemistry, vol. 10, no. 8, pp. 658-674, 1905/01/01 1905.

[48] S. Byrnes, G. Thiessen, and E. Fu, "Progress in the development of paper-based diagnostics for lowresource point-of-care settings," (in eng), Bioanalysis, vol. 5, no. 22, pp. 2821-2836, 2013.

[49] R. Lucas, "Ueber das Zeitgesetz des kapillaren Aufstiegs von Flüssigkeiten," Kolloid-Zeitschrift, vol. 23, no. 1, pp. 15-22, 1918/07/01 1918.

[50] E. W. Washburn, "The Dynamics of Capillary Flow," Physical Review, vol. 17, no. 3, pp. 273-283, 03/01/ 1921.

[51] C. H. Bosanquet, "LV. On the flow of liquids into capillary tubes," The London, Edinburgh, and Dublin Philosophical Magazine and Journal of Science, vol. 45, no. 267, pp. 525-531, 1923/03/01 1923.

[52] A. Hamraoui and T. Nylander, "Analytical Approach for the Lucas-Washburn Equation," Journal of Colloid and Interface Science, vol. 250, no. 2, pp. 415-421, 2002/06/15/ 2002.

[53] A. Hamraoui, K. Thuresson, T. Nylander, K. Eskilsson, and V. Yaminsky, "Dynamic wetting and dewetting by aqueous solutions containing amphiphilic compounds," in Surface and Colloid Science, Berlin, Heidelberg, 2001: Springer Berlin Heidelberg, pp. 113-119.

[54] N. Ichikawa and Y. Satoda, "Interface Dynamics of Capillary Flow in a Tube under Negligible Gravity Condition," Journal of Colloid and Interface Science, vol. 162, no. 2, pp. 350-355, 1994/02/01/ 1994.

[55] B. Lavi, A. Marmur, and J. Bachmann, "Porous Media Characterization by the Two-Liquid Method: Effect of Dynamic Contact Angle and Inertia," Langmuir, vol. 24, no. 5, pp. 1918-1923, 2008/03/01 2008.

[56] E. K. Rideal, "CVIII. On the flow of liquids under capillary pressure," The London, Edinburgh, and Dublin Philosophical Magazine and Journal of Science, vol. 44, no. 264, pp. 1152-1159, 1922/12/01 1922.

[57] A. Siebold, M. Nardin, J. Schultz, A. Walliser, and M. Oppliger, "Effect of dynamic contact angle on capillary rise phenomena," Colloids and Surfaces A: Physicochemical and Engineering Aspects, vol. 161 , no. 1, pp. 81-87, 2000/01/15/2000.

[58] E. Fu, S. A. Ramsey, P. Kauffman, B. Lutz, and P. Yager, "Transport in two-dimensional paper networks," (in eng), Microfluid Nanofluidics, vol. 10, no. 1, pp. 29-35, 2011/01// 2011.

[59] S. Whitaker, "Flow in porous media I: A theoretical derivation of Darcy's law," Transport in Porous Media, vol. 1, no. 1, pp. 3-25, 1986/03/01 1986.

[60] H. Darcy, Les fontaines publiques de la ville de Dijon. Dalmont, 1856.

[61] A. Koponen et al., "Permeability of Three-Dimensional Random Fiber Webs," Physical Review Letters, vol. 80, no. 4, pp. 716-719, 01/26/ 1998. 
[62] R. Benzi, S. Succi, and M. Vergassola, "The lattice Boltzmann equation: theory and applications," Physics Reports, vol. 222, no. 3, pp. 145-197, 1992/12/01/ 1992.

[63] I. Ginzbourg and D. d'Humières, "Local second-order boundary methods for lattice Boltzmann models," Journal of Statistical Physics, vol. 84, no. 5, pp. 927-971, 1996/09/01 1996.

[64] Y. H. Qian, D. D'Humières, and P. Lallemand, "Lattice BGK Models for Navier-Stokes Equation," Europhysics Letters (EPL), vol. 17, no. 6, pp. 479-484, 1992/02/01 1992.

[65] Z. Liu et al., "Liquid wicking behavior in paper-like materials: mathematical models and their emerging biomedical applications," Microfluid Nanofluidics, vol. 22, no. 11, p. 132, 2018/11/07 2018.

[66] L. Rivas, M. Medina-Sánchez, A. de la Escosura-Muñiz, and A. Merkoçi, "Improving sensitivity of gold nanoparticle-based lateral flow assays by using wax-printed pillars as delay barriers of microfluidics," Lab on a Chip, 10.1039/C4LC00972J vol. 14, no. 22, pp. 4406-4414, 2014.

[67] J. R. Choi et al., "Polydimethylsiloxane-Paper Hybrid Lateral Flow Assay for Highly Sensitive Point-ofCare Nucleic Acid Testing," Anal Chem, vol. 88, no. 12, pp. 6254-6264, 2016/06/21 2016.

[68] B. J. Toley, B. McKenzie, T. Liang, J. R. Buser, P. Yager, and E. Fu, "Tunable-delay shunts for paper microfluidic devices," (in eng), Anal Chem, vol. 85, no. 23, pp. 11545-11552, 2013.

[69] B. M. Cummins, R. Chinthapatla, F. S. Ligler, and G. M. Walker, "Time-Dependent Model for Fluid Flow in Porous Materials with Multiple Pore Sizes," Anal Chem, vol. 89, no. 8, pp. 4377-4381, 2017/04/18 2017.

[70] S. Dharmaraja et al., Programming paper networks for point of care diagnostics (SPIE MOEMSMEMS). SPIE, 2013.

[71] R. Tang et al., "Improved Analytical Sensitivity of Lateral Flow Assay using Sponge for HBV Nucleic Acid Detection," Scientific Reports, vol. 7, no. 1, p. 1360, 2017/05/02 2017.

[72] A. W. Martinez et al., "Programmable diagnostic devices made from paper and tape," Lab on a Chip, 10.1039/COLC00021C vol. 10, no. 19, pp. 2499-2504, 2010.

[73] W. Wang, W.-Y. Wu, W. Wang, and J.-J. Zhu, "Tree-shaped paper strip for semiquantitative colorimetric detection of protein with self-calibration," Journal of Chromatography A, vol. 1217, no. 24, pp. 3896-3899, 2010/06/11/2010.

[74] A. Medina, C. Pérez-Rosales, A. Pineda, and F. J. Higuera, Imbibition in pieces of paper with different shapes. 2001, pp. 537-541.

[75] S. Mendez et al., "Imbibition in Porous Membranes of Complex Shape: Quasi-stationary Flow in Thin Rectangular Segments," Langmuir, vol. 26, no. 2, pp. 1380-1385, 2010/01/19 2010.

[76] E. Fu, T. Liang, P. Spicar-Mihalic, J. Houghtaling, S. Ramachandran, and P. Yager, "Two-dimensional paper network format that enables simple multistep assays for use in low-resource settings in the context of malaria antigen detection," (in eng), Anal Chem, vol. 84, no. 10, pp. 4574-4579, 2012.

[77] B. R. Lutz, P. Trinh, C. Ball, E. Fu, and P. Yager, "Two-dimensional paper networks: programmable fluidic disconnects for multi-step processes in shaped paper," (in eng), Lab on a chip, vol. 11, no. 24, pp. 4274-4278, 2011.

[78] B. Lutz, T. Liang, E. Fu, S. Ramachandran, P. Kauffman, and P. Yager, "Dissolvable fluidic time delays for programming multi-step assays in instrument-free paper diagnostics," (in eng), Lab on a chip, vol. 13, no. 14, pp. 2840-2847, 2013.

[79] H. Noh and S. T. Phillips, "Fluidic Timers for Time-Dependent, Point-of-Care Assays on Paper," Anal Chem, vol. 82, no. 19, pp. 8071-8078, 2010/10/01 2010.

[80] H. Chen, J. Cogswell, C. Anagnostopoulos, and M. Faghri, "A fluidic diode, valves, and a sequentialloading circuit fabricated on layered paper," Lab on a Chip, 10.1039/C2LC20970E vol. 12, no. 16, pp. 2909-2913, 2012.

[81] H. Liu, X. Li, and R. M. Crooks, "Paper-Based SlipPAD for High-Throughput Chemical Sensing," Anal Chem, vol. 85, no. 9, pp. 4263-4267, 2013/05/07 2013.

[82] C. K. W. Koo, F. He, and S. R. Nugen, "An inkjet-printed electrowetting valve for paper-fluidic sensors," Analyst, 10.1039/C3AN01114C vol. 138, no. 17, pp. 4998-5004, 2013.

[83] P. Mandal, R. Dey, and S. Chakraborty, "Electrokinetics with "paper-and-pencil" devices," Lab on a Chip, 10.1039/C2LC40681K vol. 12, no. 20, pp. 4026-4028, 2012.

[84] X. Li, P. Zwanenburg, and X. Liu, "Magnetic timing valves for fluid control in paper-based microfluidics," Lab on a Chip, 10.1039/C3LC00006K vol. 13, no. 13, pp. 2609-2614, 2013. 
[85] M. Fratzl et al., "Magnetic Two-Way Valves for Paper-Based Capillary-Driven Microfluidic Devices," ACS Omega, vol. 3, no. 2, pp. 2049-2057, 2018/02/28 2018.

[86] A. R. Rezk, A. Qi, J. R. Friend, W. H. Li, and L. Y. Yeo, "Uniform mixing in paper-based microfluidic systems using surface acoustic waves," Lab on a Chip, 10.1039/C2LC21065G vol. 12, no. 4, pp. 773779, 2012.

[87] N.-T. Nguyen and Z. Wu, "Micromixers-a review," Journal of Micromechanics and Microengineering, vol. 15, no. 2, pp. R1-R16, 2004/12/09 2004.

[88] Z. W. Zhong, R. G. Wu, Z. P. Wang, and H. L. Tan, "An investigation of paper based microfluidic devices for size based separation and extraction applications," Journal of Chromatography $B$, vol. 1000, pp. 41-48, 2015/09/01/ 2015.

[89] M. F. Mora et al., "Patterning and Modeling Three-Dimensional Microfluidic Devices Fabricated on a Single Sheet of Paper," Anal Chem, vol. 91, no. 13, pp. 8298-8303, 2019/07/02 2019.

[90] R. Urteaga, E. Elizalde, and C. L. A. Berli, "Transverse solute dispersion in microfluidic paper-based analytical devices (4PADs)," Analyst, 10.1039/C8AN00149A vol. 143, no. 10, pp. 2259-2266, 2018.

[91] A. J. P. Martin and R. L. M. Synge, "A new form of chromatogram employing two liquid phases," Biochemical Journal, vol. 35, no. 12, p. 1358, 1941.

[92] W. J. Whelan, "The advent of paper chromatography," The FASEB Journal, vol. 9, no. 2, pp. 287-288, 1995/02/01 1995.

[93] F. Sanger, "SEQUENCES, SEQUENCES, AND SEQUENCES," Annual Review of Biochemistry, vol. 57, no. 1, pp. 1-29, 1988/06/01 1988.

[94] H. G. Kunkel and A. Tiselius, "Electrophoresis of proteins on filter paper," (in eng), J Gen Physiol, vol. 35, no. 1, pp. 89-118, 1951.

[95] G. Zweig, J. R. Whitaker, and R. J. Block, Paper Chromatography and Electrophoresis: Paper chromotography by J. Sherman and G. Zweig (Paper Chromatography and Electrophoresis). Academic Press, 1971.

[96] L. Y. Shiroma, M. Santhiago, A. L. Gobbi, and L. T. Kubota, "Separation and electrochemical detection of paracetamol and 4-aminophenol in a paper-based microfluidic device," Analytica Chimica Acta, vol. 725, pp. 44-50, 2012/05/06/ 2012.

[97] R. S. Nakhal, D. Wood, C. Woodhouse, and S. M. Creighton, "False-positive pregnancy tests following enterocystoplasty," BJOG: An International Journal of Obstetrics \& Gynaecology, vol. 119, no. 3, pp. 366-368, 2012/02/01 2012.

[98] X. Li, J. Tian, and W. Shen, "Progress in patterned paper sizing for fabrication of paper-based microfluidic sensors," Cellulose, vol. 17, no. 3, pp. 649-659, 2010/06/01 2010.

[99] J. Park, J. H. Shin, and J.-K. Park, "Pressed Paper-Based Dipstick for Detection of Foodborne Pathogens with Multistep Reactions," Anal Chem, vol. 88, no. 7, pp. 3781-3788, 2016/04/05 2016.

[100] S. Ahmed, M.-P. N. Bui, and A. Abbas, "Paper-based chemical and biological sensors: Engineering aspects," Biosensors and Bioelectronics, vol. 77, pp. 249-263, 2016/03/15/ 2016.

[101] W. W. Yu and I. M. White, "Inkjet-printed paper-based SERS dipsticks and swabs for trace chemical detection," Analyst, Article vol. 138, no. 4, pp. 1020-1025, 2013.

[102] S. Di Risio and N. Yan, "Piezoelectric Ink-Jet Printing of Horseradish Peroxidase: Effect of Ink Viscosity Modifiers on Activity," Macromolecular Rapid Communications, vol. 28, no. 18-19, pp. 1934-1940, 2007/09/18 2007.

[103] M. S. Khan et al., "Biosurface engineering through ink jet printing," Colloids and Surfaces B: Biointerfaces, vol. 75, no. 2, pp. 441-447, 2010/02/01/ 2010.

[104] G. E. Fridley, H. Q. Le, E. Fu, and P. Yager, "Controlled release of dry reagents in porous media for tunable temporal and spatial distribution upon rehydration," Lab on a Chip, 10.1039/C2LC40785J vol. 12, no. 21, pp. 4321-4327, 2012.

[105] J. L. Osborn, B. Lutz, E. Fu, P. Kauffman, D. Y. Stevens, and P. Yager, "Microfluidics without pumps: reinventing the T-sensor and H-filter in paper networks," Lab on a Chip, 10.1039/C004821F vol. 10, no. 20, pp. 2659-2665, 2010.

[106] W. Dungchai, O. Chailapakul, and C. S. Henry, "Use of multiple colorimetric indicators for paperbased microfluidic devices," (in eng), Analytica chimica acta, vol. 674, no. 2, pp. 227-233, 2010/08// 2010. 
[107] L. Guan, R. Cao, J. Tian, H. McLiesh, G. Garnier, and W. Shen, "A preliminary study on the stabilization of blood typing antibodies sorbed into paper," Cellulose, vol. 21, no. 1, pp. 717-727, 2014/02/01 2014.

[108] A. W. Martinez, S. T. Phillips, E. Carrilho, S. W. Thomas, H. Sindi, and G. M. Whitesides, "Simple Telemedicine for Developing Regions: Camera Phones and Paper-Based Microfluidic Devices for Real-Time, Off-Site Diagnosis," Anal Chem, vol. 80, no. 10, pp. 3699-3707, 2008/05/01 2008.

[109] L. Liang et al., "Aptamer-based fluorescent and visual biosensor for multiplexed monitoring of cancer cells in microfluidic paper-based analytical devices," Sensors and Actuators B: Chemical, vol. 229, pp. 347-354, 2016/06/28/ 2016.

[110] X. Wei et al., "Microfluidic Distance Readout Sweet Hydrogel Integrated Paper-Based Analytical Device ( $\mu$ DiSH-PAD) for Visual Quantitative Point-of-Care Testing," Anal Chem, vol. 88, no. 4, pp. 2345-2352, 2016/02/16 2016.

[111] S. Dalirirad and A. J. Steckl, "Aptamer-based lateral flow assay for point of care cortisol detection in sweat," Sensors and Actuators B: Chemical, vol. 283, pp. 79-86, 2019/03/15/ 2019.

[112] E. Frohnmeyer et al., "Aptamer lateral flow assays for rapid and sensitive detection of cholera toxin," Analyst, 10.1039/C8AN01616J vol. 144, no. 5, pp. 1840-1849, 2019.

[113] X. Mao, Y. Ma, A. Zhang, L. Zhang, L. Zeng, and G. Liu, "Disposable Nucleic Acid Biosensors Based on Gold Nanoparticle Probes and Lateral Flow Strip," Anal Chem, vol. 81, no. 4, pp. 1660-1668, 2009/02/15 2009.

[114] B. Kavosi, R. Hallaj, H. Teymourian, and A. Salimi, "Au nanoparticles/PAMAM dendrimer functionalized wired ethyleneamine-viologen as highly efficient interface for ultra-sensitive $\alpha$ fetoprotein electrochemical immunosensor," Biosensors and Bioelectronics, vol. 59, pp. 389-396, 2014/09/15/ 2014.

[115] M. Jauset-Rubio et al., "Ultrasensitive, rapid and inexpensive detection of DNA using paper based lateral flow assay," Scientific Reports, Article vol. 6, p. 37732, 11/25/online 2016.

[116] N. Ying et al., "Lateral flow nucleic acid biosensor for sensitive detection of microRNAs based on the dual amplification strategy of duplex-specific nuclease and hybridization chain reaction," PLOS ONE, vol. 12, no. 9, p. e0185091, 2017.

[117] Y. K. Oh, H.-A. Joung, H. S. Han, H.-J. Suk, and M.-G. Kim, "A three-line lateral flow assay strip for the measurement of $\mathrm{C}$-reactive protein covering a broad physiological concentration range in human sera," Biosensors and Bioelectronics, vol. 61, pp. 285-289, 2014/11/15/ 2014.

[118] M. A. Dineva, D. Candotti, F. Fletcher-Brown, J.-P. Allain, and H. Lee, "Simultaneous Visual Detection of Multiple Viral Amplicons by Dipstick Assay," Journal of Clinical Microbiology, vol. 43, no. 8, p. 4015, 2005.

[119] Y. Chen et al., "Near-infrared fluorescence-based multiplex lateral flow immunoassay for the simultaneous detection of four antibiotic residue families in milk," Biosensors and Bioelectronics, vol. 79, pp. 430-434, 2016/05/15/ 2016.

[120] Y. Chen et al., "A dual-readout chemiluminescent-gold lateral flow test for multiplex and ultrasensitive detection of disease biomarkers in real samples," Nanoscale, 10.1039/C6NR04017A vol. 8, no. 33, pp. 15205-15212, 2016.

[121] S. Lee, S. Mehta, and D. Erickson, "Two-Color Lateral Flow Assay for Multiplex Detection of Causative Agents Behind Acute Febrile Illnesses," Anal Chem, vol. 88, no. 17, pp. 8359-8363, 2016/09/06 2016.

[122] D. Zhang et al., "Quantitative and ultrasensitive detection of multiplex cardiac biomarkers in lateral flow assay with core-shell SERS nanotags," Biosensors and Bioelectronics, vol. 106, pp. 204-211, 2018/05/30/2018.

[123] E. M. Fenton, M. R. Mascarenas, G. P. López, and S. S. Sibbett, "Multiplex Lateral-Flow Test Strips Fabricated by Two-Dimensional Shaping," ACS Applied Materials \& Interfaces, vol. 1, no. 1, pp. 124129, 2009/01/28 2009.

[124] J. P. He, N. I. Katis, W. R. Eason, and L. C. Sones, "Rapid Multiplexed Detection on Lateral-Flow Devices Using a Laser Direct-Write Technique," Biosensors, vol. 8, no. 4, 2018.

[125] T. Yonekita et al., "Development of a novel multiplex lateral flow assay using an antimicrobial peptide for the detection of Shiga toxin-producing Escherichia coli," Journal of Microbiological Methods, vol. 93, no. 3, pp. 251-256, 2013/06/01/ 2013. 
[126] Y. Zhao et al., "Rapid multiplex detection of 10 foodborne pathogens with an up-converting phosphor technology-based 10-channel lateral flow assay," Scientific Reports, Article vol. 6, p. 21342, 02/17/online 2016.

[127] B. Hayes, C. Murphy, A. Crawley, and R. O’Kennedy, "Developments in Point-of-Care Diagnostic Technology for Cancer Detection," Diagnostics, vol. 8, no. 2, 2018.

[128] X. Fang, S. Wei, and J. Kong, "Paper-based microfluidics with high resolution, cut on a glass fiber membrane for bioassays," Lab on a Chip, 10.1039/C3LC51246K vol. 14, no. 5, pp. 911-915, 2014.

[129] Y. Hao et al., "A naphthalimide-based azo colorimetric and ratiometric probe: synthesis and its application in rapid detection of cyanide anions," Analytical Methods, 10.1039/C3AY41931B vol. 6, no. 8, pp. 2478-2483, 2014.

[130] A. A. Weaver et al., "Paper Analytical Devices for Fast Field Screening of Beta Lactam Antibiotics and Antituberculosis Pharmaceuticals," Anal Chem, vol. 85, no. 13, pp. 6453-6460, 2013/07/02 2013.

[131] Y. Yang, E. Noviana, M. P. Nguyen, B. J. Geiss, D. S. Dandy, and C. S. Henry, "Paper-Based Microfluidic Devices: Emerging Themes and Applications," Anal Chem, vol. 89, no. 1, pp. 71-91, 2017/01/03 2017.

[132] A. Ismail et al., "Colorimetric analysis of the decomposition of S-nitrosothiols on paper-based microfluidic devices," Analyst, 10.1039/C6AN01439A vol. 141, no. 22, pp. 6314-6320, 2016.

[133] C. Sicard et al., "Tools for water quality monitoring and mapping using paper-based sensors and cell phones," Water Research, vol. 70, pp. 360-369, 2015/03/01/ 2015.

[134] S. Jain et al., "Performance of an Optimized Paper-Based Test for Rapid Visual Measurement of Alanine Aminotransferase (ALT) in Fingerstick and Venipuncture Samples," (in eng), PloS one, vol. 10, no. 5, pp. e0128118-e0128118, 2015.

[135] X. Mu et al., "A paper-based skin patch for the diagnostic screening of cystic fibrosis," Chemical Communications, 10.1039/C5CC00717H vol. 51, no. 29, pp. 6365-6368, 2015.

[136] E. Morales-Narváez, T. Naghdi, E. Zor, and A. Merkoçi, "Photoluminescent Lateral-Flow Immunoassay Revealed by Graphene Oxide: Highly Sensitive Paper-Based Pathogen Detection," Anal Chem, vol. 87, no. 16, pp. 8573-8577, 2015/08/18 2015.

[137] L.-M. Hu et al., "Advantages of time-resolved fluorescent nanobeads compared with fluorescent submicrospheres, quantum dots, and colloidal gold as label in lateral flow assays for detection of ractopamine," Biosensors and Bioelectronics, vol. 91, pp. 95-103, 2017/05/15/ 2017.

[138] S. Takalkar, K. Baryeh, and G. Liu, "Fluorescent carbon nanoparticle-based lateral flow biosensor for ultrasensitive detection of DNA," Biosensors and Bioelectronics, vol. 98, pp. 147-154, 2017/12/15/ 2017.

[139] L. A. Torre et al., "Ovarian cancer statistics, 2018," CA: A Cancer Journal for Clinicians, vol. 68, no. 4, pp. 284-296, 2018/07/01 2018.

[140] J. Hu et al., "Oligonucleotide-linked gold nanoparticle aggregates for enhanced sensitivity in lateral flow assays," Lab on a Chip, 10.1039/C3LC50672J vol. 13, no. 22, pp. 4352-4357, 2013.

[141] T. Wu et al., "Enhanced lateral flow assay with double conjugates for the detection of exosomes," Science China Chemistry, vol. 61, no. 11, pp. 1423-1429, 2018/11/01 2018.

[142] S. Song et al., "Multiplex Lateral Flow Immunoassay for Mycotoxin Determination," Anal Chem, vol. 86, no. 10, pp. 4995-5001, 2014/05/20 2014.

[143] T.-T. Tsai, T.-H. Huang, N. Y.-J. Ho, Y.-P. Chen, C.-A. Chen, and C.-F. Chen, "Development of a multiplex and sensitive lateral flow immunoassay for the diagnosis of periprosthetic joint infection," Scientific Reports, vol. 9, no. 1, p. 15679, 2019/10/30 2019.

[144] Q. Cao, B. Liang, T. Tu, J. Wei, L. Fang, and X. Ye, "Three-dimensional paper-based microfluidic electrochemical integrated devices (3D-PMED) for wearable electrochemical glucose detection," RSC Advances, 10.1039/C8RA09157A vol. 9, no. 10, pp. 5674-5681, 2019.

[145] E. Cho, M. Mohammadifar, and S. Choi, "A Single-Use, Self-Powered, Paper-Based Sensor Patch for Detection of Exercise-Induced Hypoglycemia," Micromachines, vol. 8, no. 9, 2017.

[146] G. Xiao et al., "A wearable, cotton thread/paper-based microfluidic device coupled with smartphone for sweat glucose sensing," Cellulose, vol. 26, no. 7, pp. 4553-4562, 2019/05/01 2019.

[147] S. Choi, "Powering point-of-care diagnostic devices," Biotechnology Advances, vol. 34, no. 3, pp. 321-330, 2016/05/01/ 2016. 
[148] Z. L. Wang, "Self-Powered Nanosensors and Nanosystems," Advanced Materials, vol. 24, no. 2, pp. 280-285, 2012/01/10 2012.

[149] M. Zhou, "Recent Progress on the Development of Biofuel Cells for Self-Powered Electrochemical Biosensing and Logic Biosensing: A Review," Electroanalysis, vol. 27, no. 8, pp. 1786-1810, 2015/08/01 2015.

[150] G. B. Stefano and R. M. Kream, "The Micro-Hospital: 5G Telemedicine-Based Care," (in eng), Med Sci Monit Basic Res, vol. 24, pp. 103-104, 2018. 